\title{
Werner Bauch als Gestalter des Campus der TH Dresden in den 1950ER JAHREN
}

\author{
Nora Kindermann
}

$\mathrm{M}$ it dem Wiederaufbau des Campus waren in den 1950er Jahren zahlreiche Professoren der damaligen Technischen Hochschule Dresden befasst. Während die neuen Hochschulbauten zumeist von den mit Lehrauftrag gebundenen Architekten geplant und gebaut wurden, lag die Konzeption und Ausführung der Freiflächen in den Händen des Landschaftsarchitekten Werner Bauch.

Der 1902 in Plauen im Vogtland geborene Bauch entstammte einer alten Gärtnerfamilie. Nach einer gärtnerisch-landwirtschaftlichen Ausbildung studierte er ab 1926 Gartenbautechnik an der Staatslehranstalt für Gartenbau in Pillnitz und schrieb sich Ende der 1920er Jahre als Gasthörer für Architektur bei Paul Schmitthenner an der TH Stuttgart ein. Seit 1930 war er als freischaffender Garten- und Landschaftsgestalter in Jößnitz bei Plauen tätig. Zunächst gestaltete er vor allem Privatgärten. In den Jahren 1938-1942 kamen weitere Tätigkeitsfelder wie Friedhöfe, Bauernhöfe, Kleingartenanlagen, Freizeitanlagen, Spielplätze, Fabrikgelände und Kuranlagen hinzu. Einer der Schwerpunkte seiner Arbeit lag zu dieser Zeit in der Tätigkeit als Landschaftsanwalt. Die Beschäftigung mit diesem Thema führte später zu weiteren Arbeiten auf dem Gebiet der Ingenieurbiologie und der Landschaftsgestaltung. Nach 1945 führte Bauch sein Planungsbüro weiter und wurde dabei seit Mitte 1948 von Werner Oppe unterstützt. Die Projekte umfassten Trümmerbegrünungen, Grünplanungen für Schulen, Kindergärten, Krankenhäuser, Friedhöfe, Volksund Kulturparks sowie landschaftsplaneri- sche Aufgaben. 1950 arbeitete er an dem Forschungsprojekt "Landschaftsdiagnose der DDR« mit und erhielt im gleichen Jahr einen Lehrauftrag als Dozent für Gartenund Landschaftsgestaltung an der TH Dresden beim Institut für Städtebau. In diese Zeit fallen auch die ersten Planungen, die Bauch für die Freiflächengestaltung der Universitätsgebäude der TH Dresden anfertigte. 1952 erfolgte die Ernennung zum Professor, 1955 wurde er Leiter des neu eingerichteten Lehrstuhls für Gartenkunst, Landschaftsgestaltung und Ingenieurbiologie. 1957 zog Bauch nach Dresden, ein Jahr später folgte ihm sein Mitarbeiter Werner Oppe mit dem Entwurfsbüro. 1963 erhielt Bauch den Ehrendoktortitel der Hochschule für Garten- und Weinbau in Budapest. Im Jahr 1968 beendete er seine Arbeit an der TH Dresden und emeritierte. Werner Bauch starb am 12.05.1983 in Dresden. ${ }^{1}$

Nach eigener Aussage wurde Bauch vor allem durch die Jahre an der TH Stuttgart geprägt. ${ }^{2}$ Dort herrschte die Idealvorstellung, dass Architekt und Landschaftsarchitekt eng zusammen arbeiteten, Garten und Gebäude untrennbar zueinander gehörten und der Grundriss des Gebäudes keine starre Grenze mehr darstellte. Der umgebende Garten wurde über die Fenster in die Räume hinein geholt, was im Wohnbereich sicher einfacher zu verwirklichen war, aber von Bauch auch bei den Hochschulgebäuden versucht wurde. $\mathrm{Zu}$ nennen sind hier u.a. die südlichen Gärten am Willersbau, die als reine Schaugärten gedacht waren und nicht durch Wege erschlossen wurden, aber auch der Innenhof der alten Mensa (Abb. 1), 
der nahezu fließend in die mit bodentiefen Fenstern ausgestatten Räume übergeht. Diese mustergültige Gestaltung war auch als Anschauungsbeispiel für die Studierenden gedacht, sie sollten auf diesem Wege sowie »in Vorlesung und Übung das Verständnis für die Beziehungen zwischen Baukörper und Freifläche, zwischen Innenraum und Außenraum $\aleph^{3}$ erhalten.

Als gartenarchitektonische Vorbilder sah Bauch vor allem Otto Valentien, Hermann Aldinger und Hermann Mattern. Aber auch die Begegnung mit Alwin Seifert prägte seine Entwurfshaltung nachhaltig, sodass "dessen Einflüsse auf Bauchs gesamte Arbeit deutlich ablesbar sind «. ${ }^{4}$ Dieser Einfluss äußert sich in erster Linie in Seiferts "Auffassungen von Pflanzenverwendung, Garten und Landschaftsgestaltung als auch [in Hinblick] auf die von ihm geschätzte >Stuttgarter Schule der Architekten.$^{5}$ Was die Gestaltungsweise Bauchs über die Jahre seiner Entwurfstätigkeit betrifft, so stellt Rindt fest, dass sich diese

»in den 50er Jahren bis auf einige Details nicht so grundlegend von früheren Arbeiten unterscheidet, also einen recht sicheren Stil dokumentiert. [...] Die Arbeiten Bauchs der 60er Jahre zeigen doch einen Wandel in der Gestaltung, der sicher auch mit den veränderten Aufgaben der Landschaftsarchitektur zusammenhängt. So stehen jetzt Fragen der Standardisierung und Typenprojektierung von Freiraumelementen und -ausstattungen an. ${ }^{6}$

Dieser Wandel in Bauchs Entwurfsauffassungen seit den 1960er Jahren wird auch von anderer Seite bestätigt. Fibich und WolschkeBulmahn konstatieren darüber hinaus einen veränderten Materialeinsatz, der sich in erster Linie in der Verwendung von Waschbeton äußert. ${ }^{7}$ Bauch selbst schrieb zu diesem Thema, dass »in der Verwendung farbiger Bodenbeläge in Form von Kies oder Makadam, Kleinpflaster, Kieselmosaik oder Kunststeinbelägen" ein besonderer Reiz gerade im Bezug auf die Gliederung von
Freiflächen liege. ${ }^{8}$ Diese Einschätzung hat auch bei der Gestaltung der Außenanlagen auf dem Campus eine Rolle gespielt. So wurde u.a. am Willersbau der Großteil der befestigten Flächen in den Gartenhöfen mit Betonplatten ausgelegt. Ebenso erhielt der Vorplatz des Andreas-Schubert-Baus (Abb. 2) einen Belag aus großformatigen, roten und weißen Betonplatten. Über ihre Farbigkeit und Oberflächenstruktur lassen sich die Platten optisch gut voneinander unterscheiden und ergeben im Wechselspiel miteinander eine graphische Wirkung.

Seine generelle Gestaltungsabsicht für die Freiflächen an den Institutsgebäuden fasst Bauch selbst in folgende Worte: "Solche Freiflächen dienen somit neben ihren Verkehrs- und Wirtschaftsfunktionen besonders der Freizeitentspannung in den Arbeitspausen der Studenten, Angestellten und Wissenschaftler. Sie dienen ferner bis zu einem gewissen Grad einer angemessenen Repräsentation an öffentlichen Bauten, wodurch ein relativ höherer Aufwand an Gestaltungsmitteln gerechtfertigt ist. «" Sehr augenscheinlich wird diese Forderung bei der Gestaltung der Außenflächen am Potthoffbau (Abb. 3). Hier wird eine klare Zonierung der Grünfläche deutlich. Neben dem reinen Verkehrsraum mit der erforderlichen Zuwegung zu den Eingängen gibt es nahe am Gebäude einen aufwändig gestalteten Bereich mit Rosen, Stauden und Ziersträuchern, wo Sitzmöglichkeiten zum Verweilen einladen. Die erforderlichen Ruhe- und Sitzplätze sollten nach Bauchs Maßgabe »in Sonnen-, wie auch in Schattensituationen genutzt werden können. Bei diesen Erholungseinrichtungen, sowie auch vor den Fenstern der Arbeitsräume, ist es vor allen Dingen angebracht, durch Konzentration der Gestaltungsmittel zu wirken, also durch Bodenformung und Mauerbildung, durch Staudenpflanzungen, Blütenschmuck und interessante Solitärgehölze. « ${ }^{10}$ Dennoch war es ihm bei der Gestaltung von Freianlagen gerade auf dem Campus ein Hauptanliegen eine »möglichst große Einfachheit in den 
AHA!

Miszellen zur Gartengeschichte und Gartendenkmalpflege

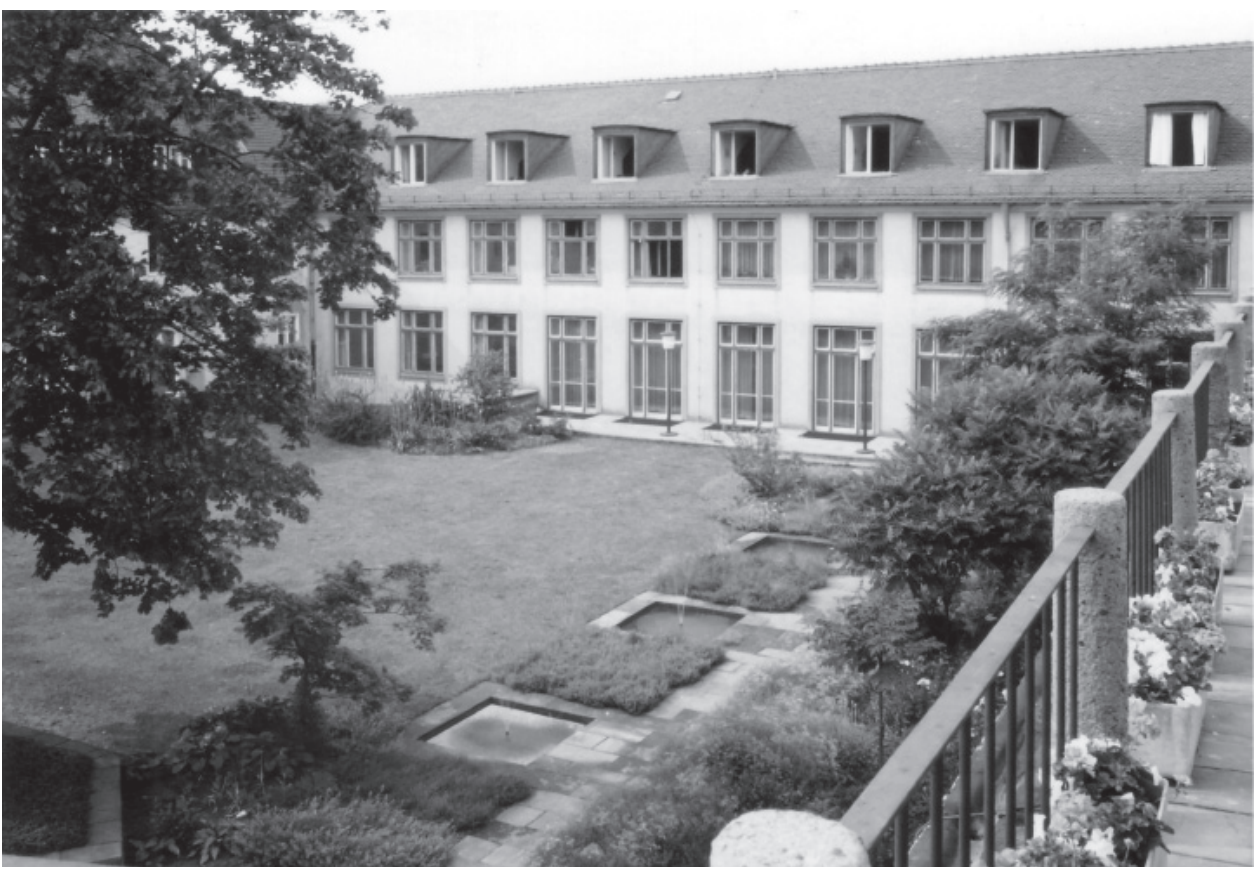

1 | Blick von der Dachterrasse in den westlichen Innenhof der Alten Mensa, 1960er Jahre. Über die quadratischen Wasserbecken an den Sitzplätzen geht der Blick über die leicht ausmodellierte Rasenfläche zu den bodentiefen Fenstern des heutigen Verwaltungstrakts (Diasammlung am Institut für Landschaftsarchitektur der TU Dresden).

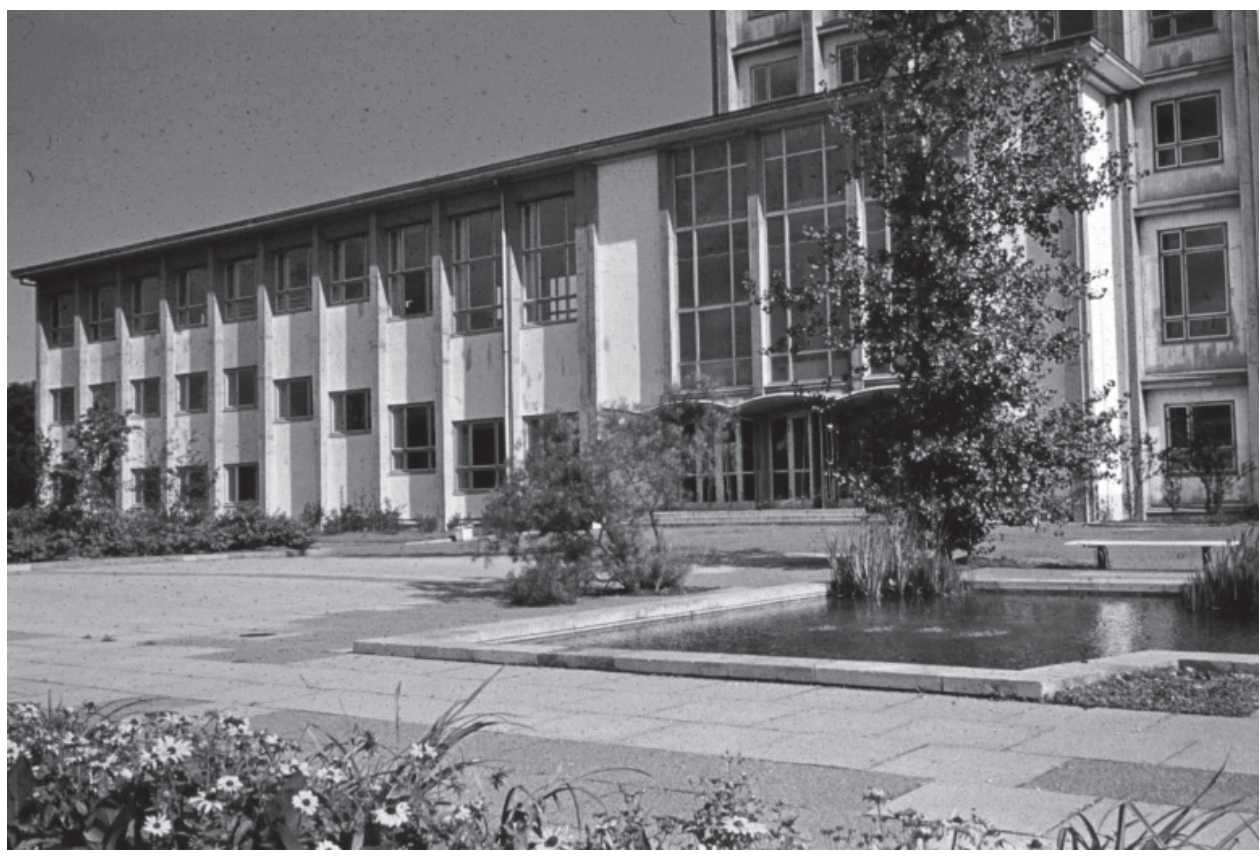

2 | Der Vorplatz des Andreas-Schubert-Baus zeigte neben einem Wasserbecken und einer leuchtenden Bepflanzung mit Rudbeckien, die typische Verwendung großformatiger Betonplatten mit unterschiedlicher Farbigkeit, wodurch die Fläche eine grafische Wirkung erhielt, 1960er Jahre (Diasammlung am Institut für Landschaftsarchitektur der TU Dresden). 
Grundformen zu finden «. ${ }^{11}$ Dieser Grundsatz wurde von anderer Seite auch als Handschrift Bauchs tituliert. Demnach zeichneten sich seine Planungen durch »Ruhe und Einfachheit der Komposition, sparsame, aber wirkungsvolle Akzentuierung und [...] Verzicht auf kurzlebig-modische Effekte« aus. ${ }^{12}$ Die Zurückhaltung hinsichtlich der baulich-gestalterischen Mittel im Freiraum sah Bauch darin begründet, dass die Pflanze einer der wichtigsten Faktoren ist, »die über Wert oder Wertlosigkeit eines Gartens bezüglich seiner Gestaltungselemente entscheiden [...]. Das Pflanzgut bildet durch den ihm eigenen Habitus letztlich ganz von selbst die interessanteste Form heraus. ${ }^{13}$ Die genannten Prinzipien lassen sich noch heute in den aus seiner Hand erhaltenen Gartenanlagen auf dem Campus der TU Dresden ablesen, die zumeist eine sehr klare und einfache Formensprache mit wenigen gliedernden baulichen Elementen aufweisen.

Im Archiv der TU Dresden sind im Nachlass von Bauch viele der Entwürfe erhalten, die er während seiner Zeit als freischaffender Landschaftsarchitekt und später während seiner Lehrtätigkeit als Professor verfasst hat. Dabei lassen sich zwischen seinen Hausgartenplanungen $^{14}$ aus den 1930er und 1940er Jahren durchaus Parallelen zu den introvertierten, oftmals auf drei Seiten von Gebäuden umschlossenen Gartenbereichen auf dem Campus ziehen. So ist die Thematik des Wasserbeckens wie sie z.B. im Senkgarten des Barkhausenbaus (Abb. 4) zu finden ist, in Form von Schwimm- und Pflanzenbecken in fast allen Hausgartenanlagen Bauchs enthalten. Es sind in allen Fällen rechteckige Beckenformen, die meist in Terrassenflächen integriert sind. Auch hinsichtlich der Verlegeart der Platten kann man Analogien finden, so liegen am Gebäude die Platten eng nebeneinander und sind über Mörtelfugen zu einer Fläche verbunden. Führt der Weg in den Gartenraum hinein, verspringen die Platten und bilden keine gerade Wegekante mehr aus. Dadurch wird eine Verz- ahnung mit den angrenzenden Staudenbeeten beziehungsweise dem Rasen erreicht, die zum Teil durch größere Fugen, in denen ebenfalls Gras wächst, noch gesteigert wird.

Dabei ist Bauch durchaus ein Kind seiner Zeit und wendet sich wie viele andere Landschaftsarchitekten dieser Zeit von den bisherigen verspielten Formen ab, hin zu klaren Kanten und einer an der Architektur orientierten Gartengestaltung, wie es u.a. Reich mit dem Satz: »Ich liebe nicht die künstliche Unordnung, könnte auch sagen, manche mögen schräge und amorphe Formen, ich nicht mehr. « forderte. ${ }^{15}$ Die Rückbesinnung auf eine klare, architektonische Form hatte ihre Ursache auch in den neuen Werkstoffen, wie zum Beispiel dem Beton, die bedingt durch ihre industrielle Fertigung genormte Formen aufwiesen und eine architektonische Bauweise erleichterten. ${ }^{16}$ Auch der Punkt, dass bei der Verwendung klarer Formen diesen immer ein Gegenpol in Form von freiwachsenden Pflanzen gegenüberzustellen sei, war Konsens zahlreicher Landschaftsarchitekten der Zeit. ${ }^{17}$ So formulierte Otto Valentien schon 1958: "Der Bau von Weg und Mauer, die ganze Gliederung des Gartens [...] erfolgt nach architektonischen Gesetzen. Der Pflanze belassen wir jedoch als Gegenpol ihre volle Natürlichkeit und streuen sie locker und zwanglos in den geometrischen Grundriß ein. $\aleph^{18}$ Diesem Grundsatz folgte auch Bauch, der innerhalb der Staudenpflanzungen Wert auf eine gruppenhafte und flächige Verwendung der Stauden legte, wodurch Ruhe im Grundgerüst entstand und die Einzelpflanze »nur bei besonderer Formenschönheit oder interessantem Wuchs im Wechselspiel und als Betonung des Standorts" zur Anwendung kam. ${ }^{19}$ Die Pflanzung sollte mit einer Kombination aus Blatt- und Blütenstauden sowie Gehölzen das ganze Jahr über interessante Aspekte bieten. Hinsichtlich der Pflanzenverwendung orientierte er sich damit voll am Zeitgeist, wobei der Fokus auf der Verwendung von Blattschmuckstauden wie Riesenbärenklau (Heracleum mantegaz- 
AHA!

Miszellen zur Gartengeschichte und Gartendenkmalpflege

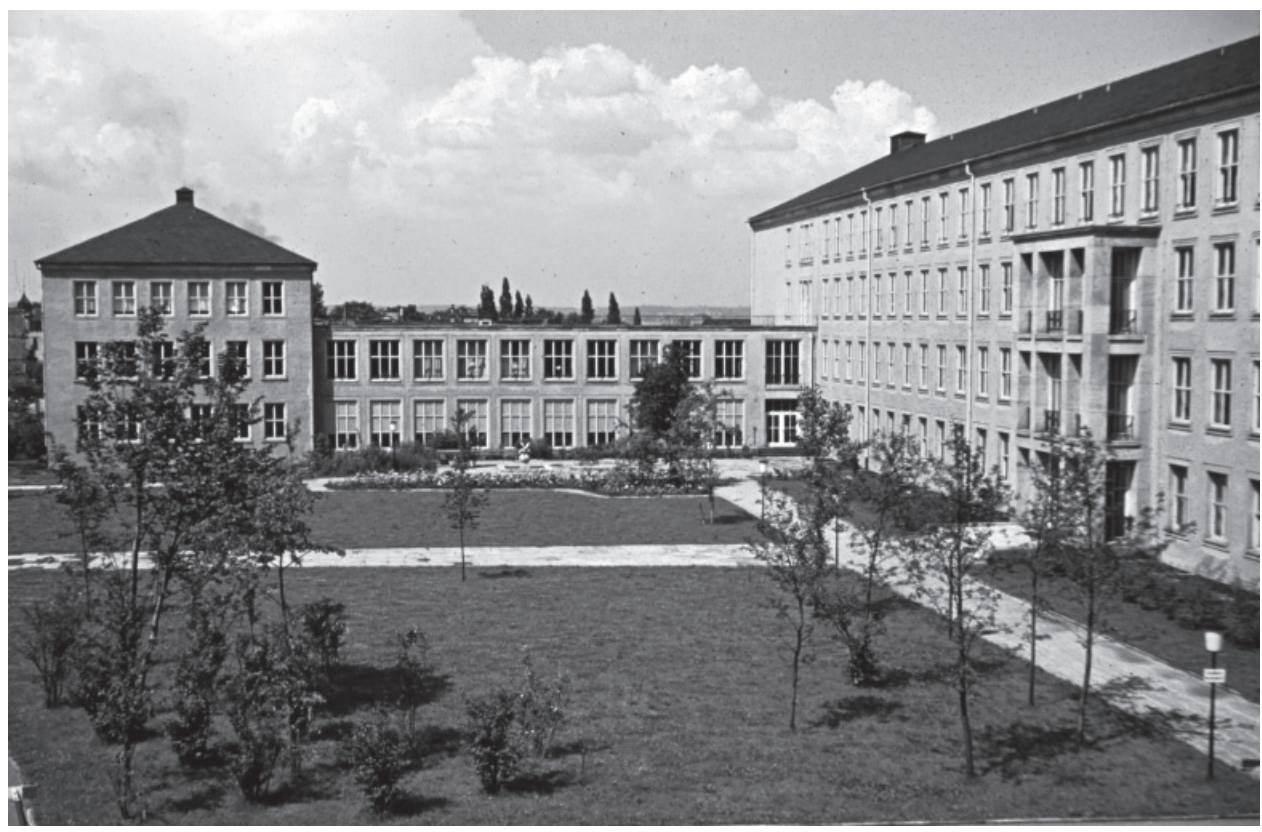

3 | Das historische Foto des Potthoffbaus zeigt deutlich die Zonierung der Grünflächen mit stark ausgestalteten und mit Stauden, Rosen und Ziersträuchern akzentuierten Aufenthaltsbereichen sowie den eher unter praktischen Gesichtspunkten mit relativ einfachen Mitteln gestalteten Umgebungsbereichen des Institutsgebäudes, 1960er Jahre (Diasammlung am Institut für Landschaftsarchitektur der TU Dresden).

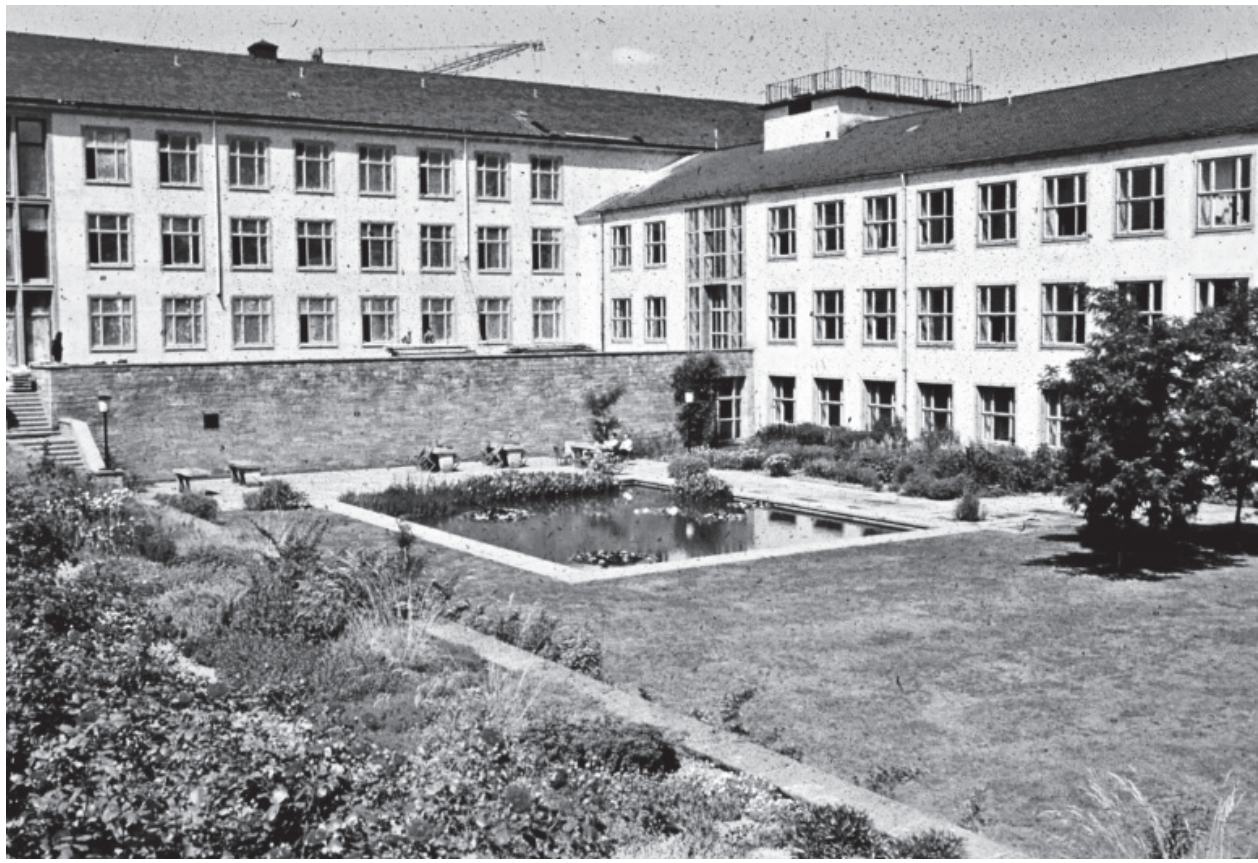

4 | Blick in den Senkgarten des Barkhausenbaus. Das große Wasserbecken dominiert die untere Gartenebene. Die nördlichen Terrassenebenen waren ursprünglich üppig mit Stauden und Rosen bepflanzt, 1960er Jahre (Diasammlung am Institut für Landschaftsarchitektur der TU Dresden). 
zianum), Weidenblättriger Sonnenblume (Helianthus salicifolius) und Federmohn (Macleya cordata) lag. Was die Gehölze betrifft, so verwendete er neben bizarr wachsenden Arten wie Essigbaum (Rhus typhina) und Aralie (Aralia elata) auch solche mit Rindenfärbung wie verschiedene CornusArten. Bei den Blütengehölzen bevorzugte er gefüllt blühende Formen wie Kerrien (Kerria japonica 'Pleniflora').

Die von Fibich und Wolschke-Bulmahn gewonnene Erkenntnis, dass »Bauch und Oppe [...] architektonische, handwerklich hochwertig ausgeführte Gestaltungsmittel wie Natursteinmauern, Pergolen, Wasserbecken und Freitreppen zu freien Pflanzungen von hoher Artenvielfalt in Kontrast $\aleph^{20}$ setzten, kann auch für den Campus der TU Dresden bestätigt werden, wobei es hier vor allem wiederum der Senkgarten am Barkhausenbau, die nördlichen Gartenhöfe am Willersbau, aber auch der Südosthof des Hülssebaus sind, die noch heute eine Kombination all dieser Gestaltungsmittel zeigen.

Als Fazit kann Werner Bauch nochmals zitiert werden: »Bei der Vielzahl der locker im Freiraum stehenden Hörsaal- und Institutsbauten kann die Variationsfülle gestalterischer Mittel, besonders auch pflanzlicher Möglichkeiten, voll ausgeschöpft werden. $\ll^{21}$ Dies ist ihm auf dem Campus der TU Dresden in besonderem Maße gelungen und es ist höchste Zeit die noch vorhandenen gestalterischen Qualitäten wert zu schätzen, zu erhalten und wieder in voller Schönheit erblühen zu lassen. Aus diesem Grund entstanden im Sommersemester 2014 an der Professur für Geschichte der Landschaftsarchitektur zwei studentische Arbeiten über die Außenanlagen des Willers- und des Hülssebaus, die gartendenkmalpflegerische Leitlinien für den künftigen Umgang aufzeigen und die im Folgenden zusammengefasst sind.
1 Rindt, Christiane: Werner Bauch- Leben und Werk im Überblick, in: Institut für Umweltgeschichte und Regionalentwicklung e.V (Hg.): Landschaft und Planung in den neuen Bundesländern Rückblick, Berlin 1999, S. 153-184. Vergleiche hierzu auch Gröning, Gert; Wolschke-Bulmahn, Joachim: Grüne Biographien - Biographisches Handbuch zur Landschaftsarchitektur des 20. Jahrhunderts in Deutschland, Berlin Hannover 1997, S. 28-29.

2 Die "Stuttgarter Schule» lehnte zwar den Historismus ab, verfolgte aber eine heimatverbundene und traditionalistische Architekturauffassung. Neu entstehende Bauwerke sollten in einer material- und werkgerechten Bauweise konstruiert und in handwerklichen Traditionen mit natürlichen Materialien ausgeführt werden. Mit diesen Grundsätzen bildete die Stuttgarter Schule einen Gegenpol zur Architektur des Bauhauses.

3 Bauch, Werner: Freiflächengestaltung an Kultur- und Institutsbauten der Technischen Hochschule Dresden, in: Deutsche Gartenarchitektur, 2/1960, S. 47-54, hier: S. 54.

4 Rindt 1999, S. 156.

Ebd.

Ebd., S. 168-169

7 Fibich, Peter; Wolschke-Bulmahn, Joachim: Werner Bauch - Landschaftsarchitekt in zwei politischen Systemen, in: Stadt + Grün, 1/2006, S. 20-24, hier: S. 23.

8 Bauch 1960, S. 53.

9 Ebd., S. 49.

10 Ebd., S. 53.

11 Ebd., S. 53

12 Linke, Harald: Prof. Dr. h. c. Werner Bauch 65 Jahre alt, in: Deutsche Gartenarchitektur, 1/1967, S. 90.

13 Bauch 1960, S. 53.

14 Im Archiv der TU Dresden gibt es Akten, Pläne und Pflanzlisten zu den Hausgartenplanungen Werner Bauchs sowie Fotos ausgeführter Anlagen. Es handelt sich dabei um folgende Findbuch-Nummern: $6,8,15,16,17,97,105,132,136,153$. Daneben wurden einige lose Pläne gesichtet, die zum Zeitpunkt der Bearbeitung (2009) noch nicht archiviert waren.

15 Reich, Alfred: Entwicklung zu klaren Formen?, in: Garten und Landschaft, 1/1962, S. 14-16, hier: S. 14.

16 U.a. Gollwitzer, Gerda; Weber, Hans Jürgen: Entwicklung zu klaren Formen, in: Garten und Landschaft, 4/1962, S. 97-103, hier S. 97.

17 U.a.: Luz, Hans: Gedanken zu den Planungen der letzten Zeit, in: Garten und Landschaft, 6/1964, S. 200. Mueller, Wolfgang; Lau, Wolfhart: Entwicklung zu klaren Formen, in: Garten und Landschaft, 4/1962, S. 97-103, hier S. 98.

18 Valentien, Otto: Der Garten am Haus, Berlin 1958, S. 5.

19 Bauch 1960, S. 53.

20 Fibich/ Wolschke-Bulmahn 2006, S. 23.

21 Bauch 1960, S. 53. 